\title{
Mechanoreceptors, feeding behaviour and trypanosome transmission in Glossina
}

\author{
D. H. Molyneux ${ }^{1}$ and L. Jenni ${ }^{2}$ \\ ${ }^{1}$ Dept. of Biology, University of Salford, Salford, M5 4WT, UK \\ ${ }^{2}$ Swiss Tropical Institute, CH-4051 Basle, Switzerland
}

Scanning electron microscope (SEM) studies of salivarian trypanosomes of the subgenera Duttonella, Nannomonas and Trypanozoon in the labrum of the tsetse fly Glossina $m$. morsitans have been carried out. These studies have shown that the colonies (rosettes) of Trypanosoma (D.) vivax and $T$. (N.) congolense occur in the proximal third of the labrum where groups of sensory receptors (sensilla) responsible for monitoring the blood flow rate occur. RICE et al. (1973) have described these sensory hairs as LCl mechanoreceptors on structural grounds; 25 to 30 such sensilla are found in each labrum. They have a basal collar and emerge from discreet pits (Figs. 1, 2). The receptor hairs extend across the food canal and, according to RICE et al. (1973), "are certain to be stimulated by the passage of food during ingestion".

SEM studies of $T$. (N.) congolense (Fig. 2) (Molyneux et al., 1979) and T. (D.) vivax (Fig. 3) (Molyneux, 1980) in the labrum of $G . m$. morsitans have demonstrated the close association between the colonies of parasites and these mechanoreceptors. The rosettes of both these subgenera are known to be attached to the labral wall (VICKERMAN, 1973; Evans et al., 1979) and the extent of rosette formation and colonization of the labrum was illustrated by CLARKE (1965) and HOARE (1972). SEM studies show that the rosettes, whilst firmly attached, are in association with the LC1 sensilla in the proximal part of the food canal. The parasites in this region are frequently attached around the base of the sensilla and sensilla hairs are entangled in the rosettes. RICE et al. (1973) reported LCl mechanoreceptors to be "fluid flow meters" which, if they receive insufficient stimulus would activate probing and pre-feeding behaviour. We suggested (MoLyneux et al., 1979) that the presence of colonies of parasites in the labrum may effect probing behaviour.

This hypothesis was confirmed by JENNI et al. (1980) who observed that $G$. m. morsitans infected with $T$. (T.) brucei (ST1B 247) probed more frequently and fed more voraciously than uninfected flies. JENNI et al. (1980) starved 40 fiies infected with $T$. (T.) brucei and 40 uninfected flies for 24 hours and 48 hours. After 24 hours, only three of the 40 infected flies offered a blood meal on mice fed on the first probe and required a mean of $5.08( \pm 0.40)$ probes before engorgement, and all 40 flies engorged. After 48 hours starvation of the infected flies, only four engorged after the first probe and the mean number of probes before feeding was $4.68(\therefore 0.37)$ and, again, all 40 flies in the batch fed. The 40 uninfected flies which were subjected to the same conditions behaved as follows : 28 fed after 24 hour's starvation, $13 \mathrm{fed}$ on the first probe and the mean number of probes before feeding was $1.89( \pm 0.21)$; after 48 hours, starvation 38 out of $40 \mathrm{fed}, 24$ on the first probe, and the mean number of probes before engorging took place was $1.53( \pm 0 \cdot 13)$. The flies used in this experiment were 20 to 35 days old and had fed as tenerals on an infected Swiss mouse.

Ten flies infected with $T$. (T.) brucei (5 male, 5 female) were then offered a series of mice on which to probe; if a fly failed to obtain a blood meal after withdrawing its proboscis it was offered another mouse. The ten infected flies infected a total of 38 mice after probing 41 times. The highest number of infective probes by a single fly was six before taking a blood meal (JENNI et al., 1980). Infected flies are thus capable of infecting mammals by successive probes before taking a blood meal.

Although Trypanozoon infections in Glossina are not usually associated with heavy labral infections, our SEM studies have revealed that groups of appatently procyclic trypomastigotes are found closely associated with LC1 mechanoreceptors in the labrum (Fig. 1). The parasites are seen attached to the bases of the sensilla or are entwined around them (see JENNI et al., 1980); clearly such interference could effect receptor function during probing behaviour and would explain the observed differences in feeding behaviour between $G$. $m$. morsitans infected with Trypanozoon and uninfected flies.

The food canal of Glossina represents a suitable structure on which to apply the principles of fluid mechanics. We have therefore (JENNI et al., 1980; LIVESEY et al., 1980) investigated the effects of trypanosome infections in the labrum on blood flow rate and the required increase in suction $(-\Delta p)$ to maintain the same rate of blood flow under conditions of trypanosome infection experience by Glossina.

Using known parameters and data the flow regime in the food canal of Glossina has been characterized. This is a pulsating flow characterized by a low Reynolds number and non-newtonian viscosity effects are considered. These considerations show the Hagen-Poiseuille theory is correct for the time average flow and time-averaged pressure gradient. The pressure drop or suck required to maintain the same meal flow rate would be inversely proportional to $\mathrm{D}^{-4} \quad(=$ diameter of the labrum) and directly proportional to the 


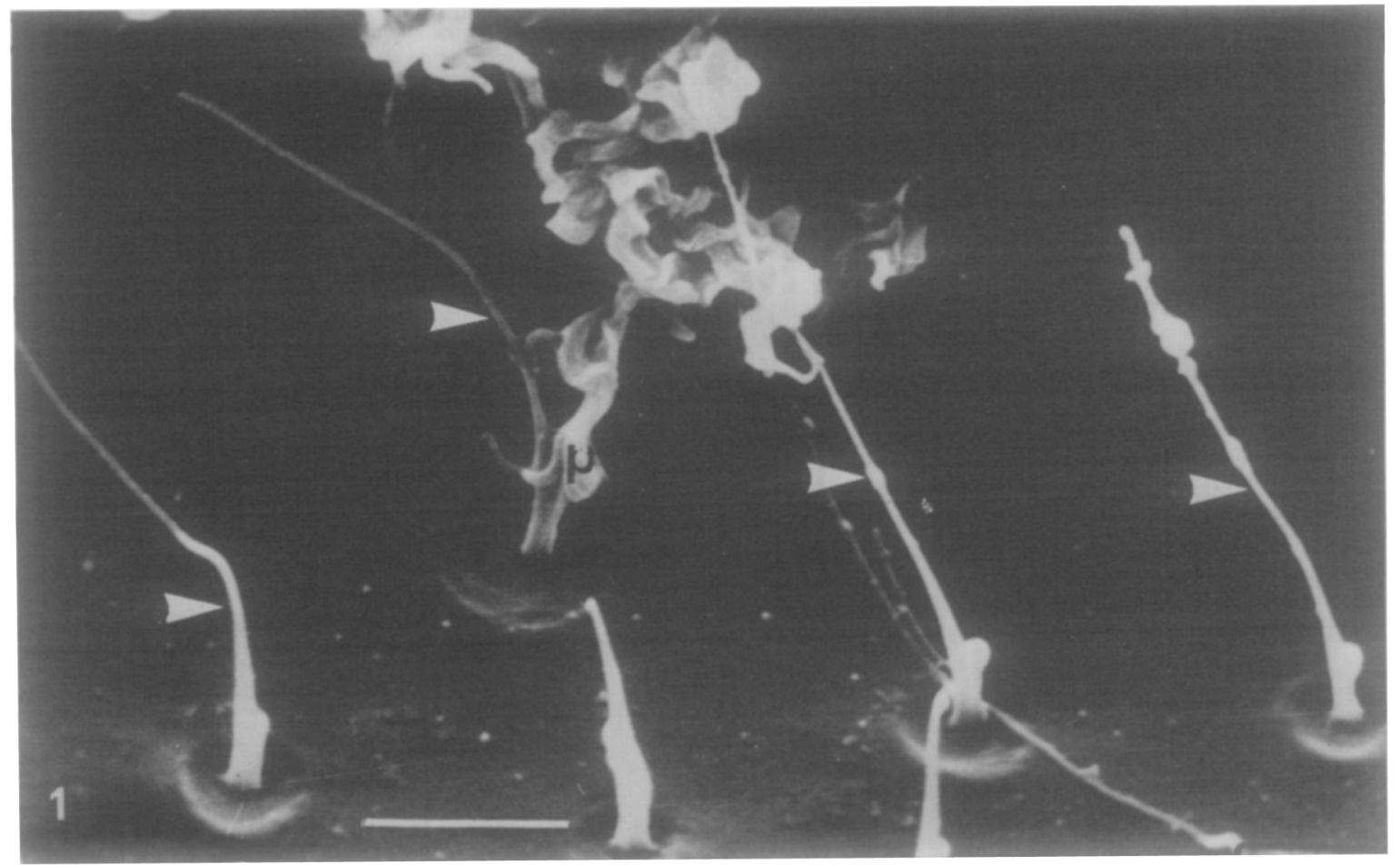

Fig. 1. Mechanoreceptors (-:arrowed) of G. m. morsitans associated with Trypanosoma (Trypanozoon) brucei trypanosomes (possibly procyclic) $(-=\mathrm{P})$ in labrum of infected fly 19 days after an infective feed. Bar $-10 \mu \mathrm{m}$.

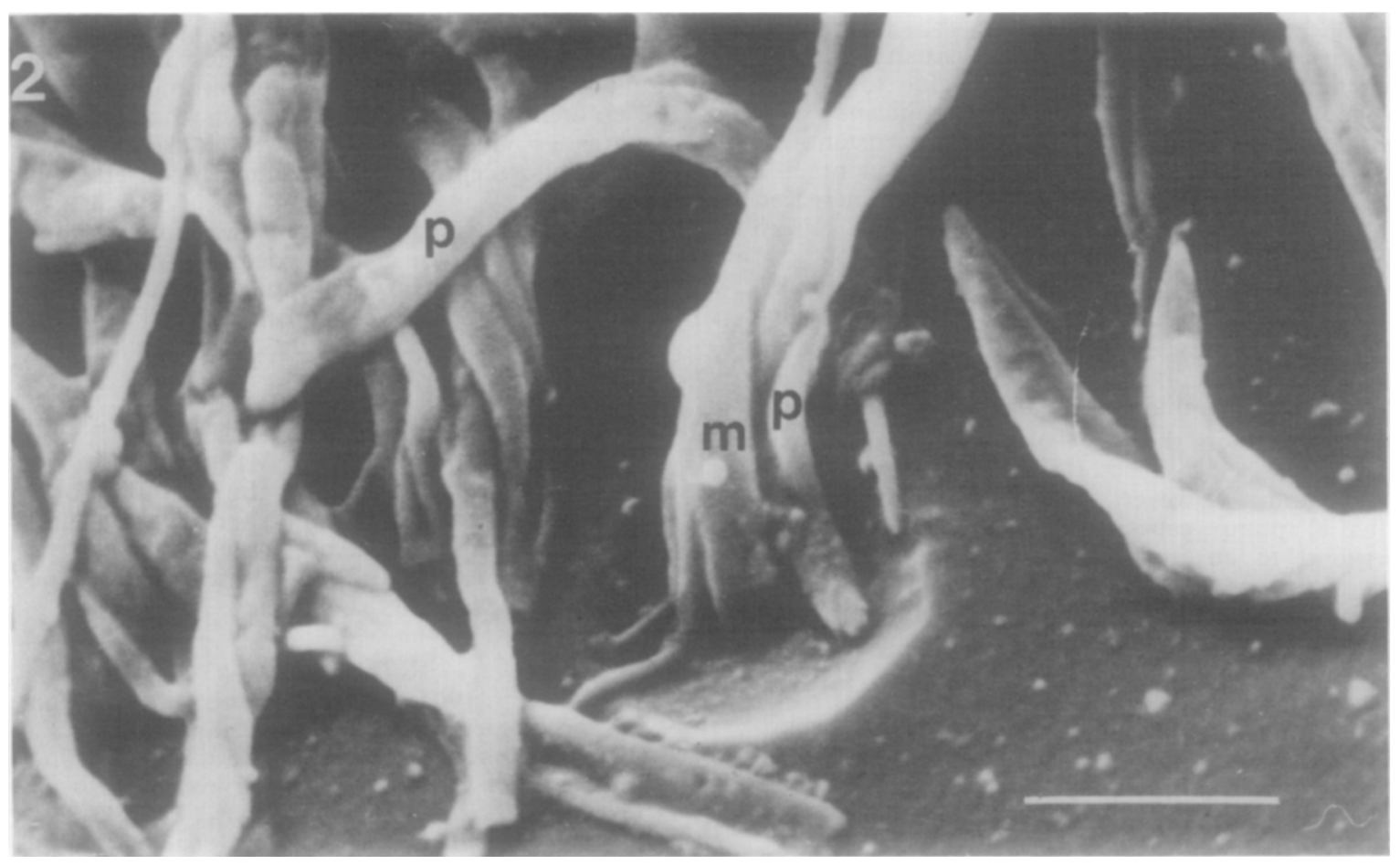

Fig. 2. Trypanosoma (Nannomonas) congolense parasites (P) associated with mechanoreceptor $(\mathrm{m})$ in labrum of $G$. m. morsitans. Bar $=4 \mu \mathrm{m}$. 


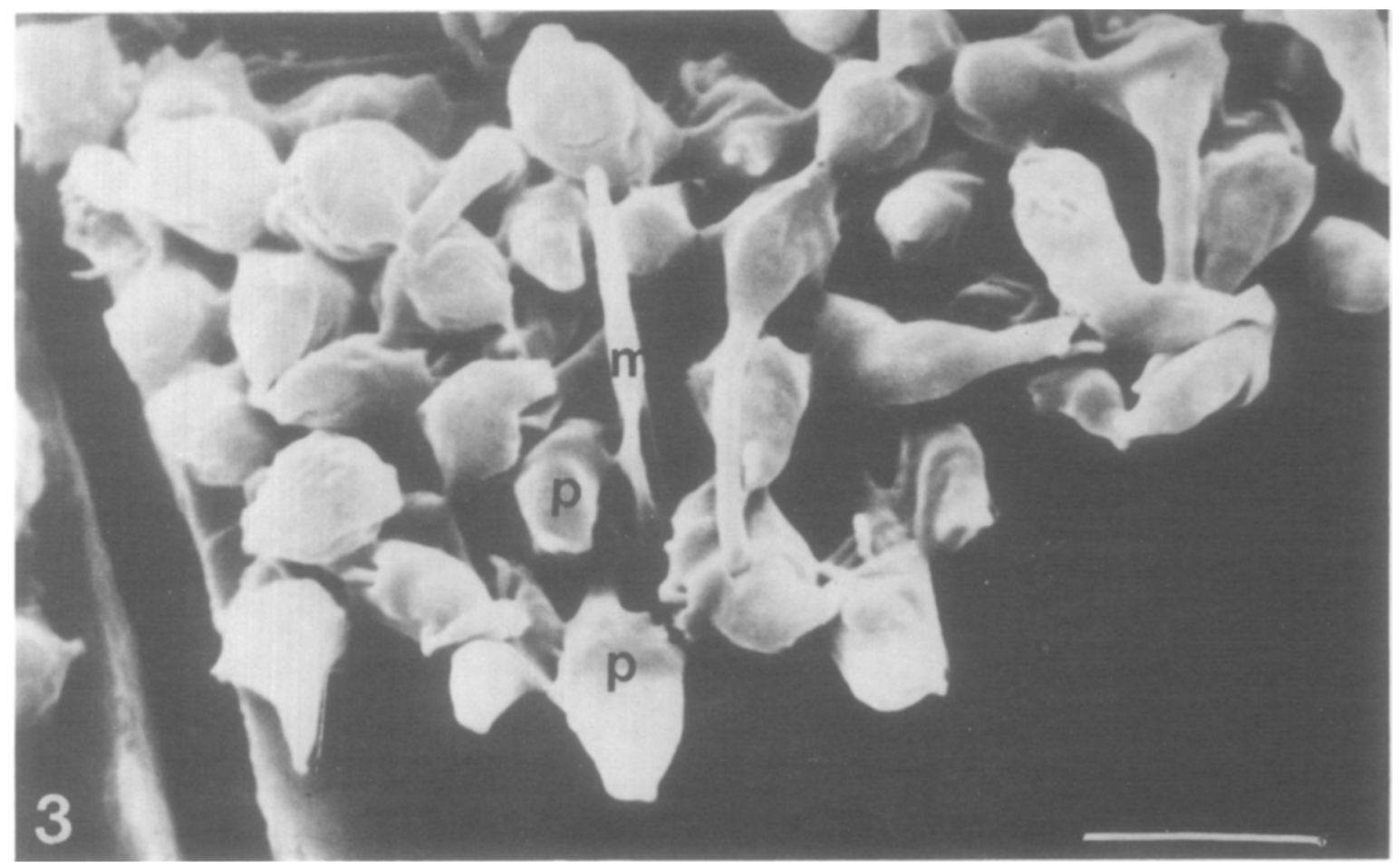

Fig. 3. Mechanoreceptor (m) emerging from a colony of $T .(D)$ vivax $(=\mathrm{P})$ in labrum. Bar $=\mathbf{4} \mu \mathrm{m}$.

All figures are scanning electron micrographs of material fixed in 2.5 ". glutaraldehyde in $0 \cdot 2 \mathrm{M}$ cacodylate buffer which were subsequently critically point dijed, nounted on double-sided sellotape on aluminium stubs, gold coated in a Polaron splutter coater and viewed in a Cambridge Steroscan IIA at accelerating voltages of $20-30 \mathrm{kV}$.

length of the labrum if the viscosity remains constant. Thus any constriction (due to trypanosome infection) could have a significant effect on flow rate if the suck remained constant or, alternatively, if the flow rate was to be maintained the pump pressure would need to be increased (JENNI et al., 1980; Livesey et al., 1980). From these calculations it can be shown that if $60^{\circ} \%$ of the length of the labrum is infected and the diameter is reduced by $50 \%$ then the factor by which flow rate would be reduced, if $-\bar{\Delta}$ is constant, is a factor of 10 (JENNI et al., 1980; LIVESEY et al., 1980). To maintain the same flow rate in the above situation the $-\Lambda_{\mathrm{p}}$ or "suck" would have to be increased by a factorof 10 also.

We know, however, that there is a limit to the cibarial pump capacity (JENNI et al., 1980) to respond to an increase in viscosity and, under such conditions, flow rate is reduced; hence the pump would have difficulty in maintaining the same flow if the labrum is heavily infected and the mechanoreceptors can be expected to detect flow rate changes (RICE et al., 1973).

We consider that evidence from the morphological and experimental studies described above, as well as theoretical fluid mechanics, demonstrate that trypanosome-mechanoreceptor interactions occur in the labrum of Glossina infected with salivarian trypanosomes and affect the feeding behaviour of infected tsetse. We suggest that the effects on mechanoreceptor feedback occur as a result of both the direct trypanosome-mechans oreceptor interactions and the effects that colonieof parasites have on How rate, which are detected by mechanoreceptors proximal to the rosettes in the labrum.

The implication can be anticipated to be relevant to the transmission and epidemiology of both human and animal trypanosomiasis transmission and epidemiology. Such considerations have already been discussed elsewhere (MOLYNEUX et al., 1979; LIVESEY et al., 1980).

\section{Acknowledgements}

We are grateful to Dr. A. M. Jordan for the supply of Glossina pupae; to the UNDP/World Bank/WHO Special Programme for Research and Training in Tropical Diseases, the UK Overseas Development Administration (Research Scheme R3495) and the Swiss NSF grant no. 3.346-0.78.

\section{References}

Clarke, J. E. (1965). Trypanosome infections in the mouthparts of Glossina morsitans Westw.: a correlation between extent of labral infection and invasion of the hypopharynx. Annals of Tropical Medicine and Parasitology, 59, 235-239.

Evans, D. A., Ellis, D. S. \& Stamford, S. (1979). Ultrastructural studies of certain aspects of the development of Trypanosoma congolense in Gloss- 
ina morsitans morsitans. Fournal of Protozoology, 26, 557-563.

Hoare, C. A. (1972). The trypanosomes of mammals: a zoological monograph. Oxford: Blackwell Scientiffic Publications.

Jenni, L., Molyneux, D. H., Livesey, J. L. \& Galun, R. (1980). Feeding behaviour of tsetse flies infected with salivarian trypanosomes. Nature, 283, 383-385.

Livesey, J. L., Molyneux, D. H. \& Jenni, L. (1980). Mechanoreceptor-trypanosome interaction in the labrum of Glossina: fluid mechanics. Acta Tropica, 37, 151-161.

Molyneux, D. H. (1980). Host-trypanosome interactions in Glossina. Insect Science and its
Applications, I, pp. 39-46.

Molyneux, D. H., Lavin, D. \& Elce, B. (1979). A possible relationship between salivarian trypanosomes and Glossina labrum mechanoreceptors. Annals of Tropical Medicine and Parasitology, 73, 287-290.

Rice, M. J., Galun, R. \& Margalit, (1973). Mouthpart sensilla of the tsetse fly and their function. III Labrocibarial sensilla. Annals of Tropical Medicine and Parasitology, 67, 109-116.

Vickerman, K. (1973). The mode of attachment of Trypanosoma vivax in the proboscis of the tsetse fly Glossina fuscipes: an ultrastructural study of the epimastigote stage of the trypanosome. Fournal of Protozoology, 20, 394-404.

\title{
Characterization of antigens from erythrocytic stages of Plasmodium falciparum reacting with human immune sera*
}

\author{
Luc H. Perrin, Renu Dayal and H. Rieder \\ WHO Immunology Research and Training Centre and Department of Medicine, Hospital Cantonal, \\ 1211 Geneva 4, Switzerland
}

Epidemiological studies and the experimental data of MCGREGOR \& WILlIAMS (1978) in the Gambia have shown that, in areas endemic for malaria, humans acquire a partial immunity to malaria infection after repeated infections, as rcflected, for example, by a decrease in the number of individuals with patent parasitaemia with age. There is evidence that humoral factors play a major role in this process. It has been shown, for example, that passive transfer of immunoglobulins from adults living in hyperendemic areas of West Africa can suppress the parasitaemia of children suffering from Plasmodium falciparum and $P$. malariae infections (COHEN et al., 1961). Some of these sera also inhibited the growth of erythrocytic forms of $P$. falciparum in vitro (WILSON \& PhILlips, 1976). There have been a number of studies on the circulating malaria antigens recognized by the serum antibodies of individuals living in endemic areas (MCGREGOR et al., 1968; WILSON et al., 1975), but little is known about the antigens of $P$. falciparum erythrocytic forms which are recognized by these sera. The aim of this work is to identify the malaria antigens present in the various erythrocytic developmental stages of $P$. falciparum and which are recognized by the sera of individuals with varying degrees of immunity.

An isolate of $P$. falciparum prepared from parasitized blood of a European patient returning from Senegal (SGE 1) was adapted to in vitro culture according to the method of TRAGER \& JENSEN (1976). The culture was synchronized twice at 34-hour intervals by plasmagel sedimentation (REESE et al., 1979) and by incubation in 5\% manitol to lyse mature erythrocytic forms (LAMBROS \& VANDERBERG, 1979). The synchronized culture showed more than $98 \%$ homogeneity for the successive developmental stages of $P$. falciparum (ring forms, trophozoites and schizonts).

Synchronized cultures were sequentially pulselabelled for four hours with ${ }^{35} \mathrm{~S}$-methionine (specific activity: $239 \mathrm{TBq} / \mathrm{mmol} ; 20 \mu \mathrm{Ci} / \mathrm{ml}$ of culture medium; $4 \%$ suspension of human RBC with $9 \%$ parasitaemia). Labelled parasitized RBC were harvested, washed twice in phosphate saline buffer and lysed in seven volumes of $0.5 \%$ NonidetP40 in Tris NaC1-EDTA buffer. The lysate was centrifuged at $20,000 \mathrm{~g}$ for $20 \mathrm{~min}$ at $4^{\circ} \mathrm{C} . \quad 85$ to $90 \%$ of the label was present in the supernatant and 8 to $15 \%$ in the pellet. The supernatant of each stage-specific culture lysate was used as antigen source of the various $P$. falciparum erythrocytic stages. The total incorporation into TCA precipitable material was respectively $0.7 \%$, $4.6 \%$ and $6.6 \%$ in ring forms, trophozoites and schizonts and less than $0.1 \%$ in normal RBC.

*Supported by WHO, the UNAP/World Bank/WHO Special Program for Research and Training in

Tropical Diseases and the Swiss National Foundation (Grant No. 3.890.0.79). 